\title{
Enhanced capability in a gas aggregation source for magnetic nanoparticles
}

\author{
Gail N. lles, ${ }^{1, a)}$ S. H. Baker, ${ }^{2}$ S. C. Thornton, ${ }^{2}$ and C. Binns ${ }^{2}$ \\ ${ }_{1}^{1}$ Institut Laue Langevin, 6 rue Jules Horowitz, 38042 Grenoble, France \\ ${ }^{2}$ University of Leicester, University Road, Leicester, LE1 7RH, UK
}

(Received 6 August 2008; accepted 4 December 2008; published online 22 January 2009)

\begin{abstract}
We describe the characterization of a high-temperature $(2000 \mathrm{~K})$ thermal gas aggregation source that is ultrahigh vacuum compatible and can cleanly deposit transition metal clusters with partial pressures of contaminants in the $10^{-11}$ mbar range allowing codeposition with highly reactive matrices. In particular, we investigate the effect of varying (i) the bath gas pressure and composition on the size distribution and flux of clusters produced and (ii) the position of the crucible within the source. The mass spectra of Fe clusters produced, recorded using a quadrupole filter, show that changing the operating conditions and configuration of the source allow a wide range of cluster sizes - 3000-320 $000 \mathrm{amu}(\sim 50-6000$ atoms for Fe or Co) to be produced. We demonstrate the cleanliness of the source by producing uncontaminated Fe clusters in rare-earth matrices. (C) 2009 American Institute of Physics. [DOI: 10.1063/1.3067761]
\end{abstract}

\section{INTRODUCTION}

The manufacture of nanocomposite thin films by depositing preformed gas-phase clusters or nanoparticles is a well established technique ${ }^{1-3}$ and is capable of producing thin films with enhanced performance in various properties. ${ }^{1-3}$ At the heart of most cluster beam sources is a region in which a supersaturated vapor of the material to be studied is generated by ejecting an atomic metal plume into a flow of a cooled inert gas. The metal vapor can be generated by sputtering, ${ }^{4-6}$ laser ablation, ${ }^{2,7,8}$ a pulsed ${ }^{9,10}$ or continuous ${ }^{11}$ arc, or thermally. ${ }^{12,13}$ A comprehensive review of cluster source design is provided in Ref. 14. The field has benefited recently from interdisciplinarity with the aerosol community leading to a better understanding of the cluster formation process and a greater ability to control it. ${ }^{15,16}$

The various methods all have their disadvantages and benefits but two features that make thermal gas aggregation sources (GASs) attractive are their inherent simplicity and cleanliness. The production of the metal vapor is similar to a molecular beam epitaxy (MBE) source and they can, in principle, be operated as cleanly. Initially a restriction with GASs was that they were restricted to relatively high vapor pressure metals but we have previously demonstrated reliable containment of the metal, without contamination, at temperatures up to $2000 \mathrm{~K}$ allowing the production of transition metal clusters. ${ }^{13}$

Since the original report ${ }^{13}$ of our ultrahigh vacuum (UHV)-compatible high-temperature thermal GAS, a number of design improvements have been made (Fig. 1). These include higher pumping speeds at the postskimmer stage enabling us to increase the size of the first aperture, the inclusion of aerodynamic lenses following the design of the Minnesota group ${ }^{17}$ and improved two-stage water and liquid nitrogen cooling tanks around the hot crucible. These have resulted in an order of magnitude increase in mass-selected cluster currents measured at the end of the mass filter

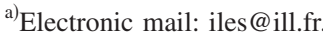

$(\sim 0.2 \mathrm{nA})$. In addition a new quadrupole mass filter has been added. It has enlarged quadrupole rods with a diameter of $25.4 \mathrm{~mm}$ and a length of $500 \mathrm{~mm}$. As well as allowing a greater throughput, the new version of the filter, operating at a frequency of $10 \mathrm{kHz}$ can size select masses as high as $350000 \mathrm{amu}$, approximately ten times higher than previously, with a resolving power $M / \Delta M$ of $\sim 70$.

The source has primarily been used to produce Fe and Co clusters and has produced films for in situ X-ray Magnetic Circular Dichroism (XMCD) studies of clean and exposed clusters on surfaces at the ESRF in Grenoble. ${ }^{18,19} \mathrm{Al}-$ though the pressure in the deposition chamber during film growth rises to $\sim 5 \times 10^{-5}$ mbar of the bath gas (usually $\mathrm{He}$ ), the combined partial pressure of the contaminant gases $-\mathrm{H}_{2} \mathrm{O}$, $\mathrm{N}_{2}$, $\mathrm{CO}$, and $\mathrm{CO}_{2}$-remains in the $10^{-11}$ mbar range. Recently its UHV performance has been put to good use to produce films of Fe nanoparticles embedded in Dy (Ref. 20) and Ho matrices.

In this paper, we report on characterization of the improved source and examine the effect of changing the bath gas composition to a mixture of $\mathrm{Ar}$ and $\mathrm{He}$ and altering their combined pressure. We also describe gas coating of the clusters in the beam by $\mathrm{H}_{2}$ and $\mathrm{N}_{2}$. In addition we investigated the effect of moving the crucible in three dimensions within the aggregation region, which reveals important information about the clustering process. Finally we report some preliminary results from Fe clusters embedded in Dy matrices, which were prepared with no detectable contamination.

\section{INSTRUMENTATION}

The incorporation of aerodynamic lenses into a cluster gas aggregation source has greatly improved the ability to produce an axisymmetric convergent-divergent particle flow. ${ }^{21,22}$ Typically nonspherical particles are subject to extra lift forces in addition to the Brownian motion and lift forces during nozzle expansion, which effectively determine the minimum width of the particle beam. The magnetic nanoparticles made by gas aggregation have been shown to have 


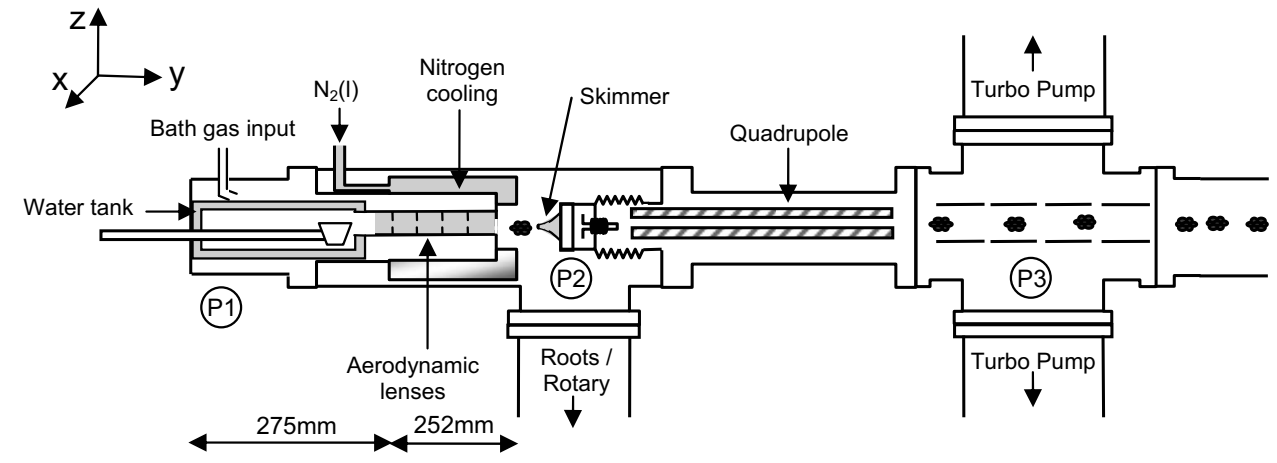

FIG. 1. GAS design improvements; two-stage water and liquid nitrogen cooling in P1 area, addition of aerodynamic lenses, increased skimmer (second aperture) diameter in P2 area, addition of turbopumps to P3 area and new quadrupole in the main chamber.

icosahedral structures ${ }^{23,24}$ and can therefore be assumed to be nonspherical however, the unique combination of aerodynamic lenses; an aperture downstream and a mass-selective quadrupole allows a particle beam of nonspherical clusters to be modeled as if it were an incompressible irrotational flow. This allows us to describe the system by the Reynolds number. In the P1 area, i.e., upstream of the lenses, the flow field can be characterized by the lens Reynold number, which relates fluid viscosity, density, and flow speed. In this case the flow field is dependent on the viscosity of helium gas and the pressure differences in the separate sections of the chamber. Calculating the gas loads at each stage provided a means to size the pumps required. After the flow field is obtained, particles are introduced and their trajectories can be calculated by integrating the equation for the local acceleration of a particle carried by a fluid flow. From this Robinson introduced the concept of the virtual particle flow field for a continuous spatial distribution of small, uniformly sized particles. $^{25}$ The model for the gas aggregation source described in this paper and the respective calculations are given a full treatment elsewhere ${ }^{26}$ however, in summary, aerodynamic focusing lenses are used to collimate the metal nanoparticle clusters (generated in the condensation chamber) into a nanoparticle stream. To get the type of flow, we used the Knudsen number equals the diameter of pipe, $d /$ mean free path, $\lambda$. If $d / \lambda>110$ this results in viscous flow, 1 $<d / \lambda<110$ results in intermediate flow and $d / \lambda<1$ results in molecular flow. The Reynolds number was used to find out if the viscous flow was turbulent or laminar and found the P1 area to be laminar, as is the case in most vacuum systems. The flow field is therefore viscous flow before the first aperture (P1), intermediate flow between the first and second apertures (P2) and molecular flow after the second aperture (P3). These changes in flow are due to the changes in mean free path.

The increased density of nanoparticles can be seen along the length of the cluster machine. By using focusing lenses, we are able to improve the efficiency of the cluster source, and generate a greater quantity of metal clusters for sample production. Evidence for the increased quantity of clusters, due to the effect of the focusing lenses, is obtained by a crystal thickness monitor (XTM) in the deposition chamber, where we are generating metal cluster deposition rates of an equivalent thickness of $0.5 \AA / \mathrm{min}$.

\section{EXPERIMENTAL RESULTS}

\section{A. Effect of varying bath gas pressure and composition}

We investigated the effect on the cluster mass spectra of changing the pressure, P1, of a pure He bath gas and also changing the proportion of Ar added to the He. Table I summarizes the associated change in mass spectra as measured by the quadrupole as a result of these gas changes. Figure 2 shows some of the spectra.

With 5 mbars of pure $\mathrm{He}(g)$ the cluster flux has increased relative to that at 3 mbars but the mass distribution remains the same as for 3 mbars (the pressure normally used). The majority of clusters have a size of $17000 \mathrm{amu}$ (290 Fe atoms) for a given power to the crucible. Adding $\operatorname{Ar}(g)$ to the bath gas changes the mass distribution. A high bath gas pressure of 12 mbars causes the peak in the mass distribution to increase to $30000 \mathrm{amu}$ (537 Fe atoms) although the increase in mean cluster size with increasing pres-

TABLE I. Data from mass spectra of Fe clusters formed in differing aggregation pressures and in varying $\operatorname{He}(g): \operatorname{Ar}(g)$ mixtures.

\begin{tabular}{ccccccc}
\hline \hline \multirow{2}{*}{ Experiment } & \multicolumn{2}{c}{ Aggregation pressure, P1 (mbar) } & \multicolumn{2}{c}{ Mass distribution (amu) } & $\begin{array}{c}\text { Intensity at peak } \\
\text { mass (nA) }\end{array}$ \\
\cline { 2 - 5 } & He & Ar & Total & Peak mass & Range & 0.04 \\
& 1 & 0 & 1 & 17000 & 50000 & 0.12 \\
& 3 & 0 & 3 & 17000 & 50000 & 0.16 \\
2 & 5 & 0 & 5 & 17000 & 50000 & 0.14 \\
& 2 & 0 & 2 & 20000 & 50000 & 0.10 \\
& 2 & 1 & 3 & 15000 & 50000 & 0.75 \\
& 2 & 2 & 4 & 12000 & 50000 & 0.02 \\
\hline \hline
\end{tabular}




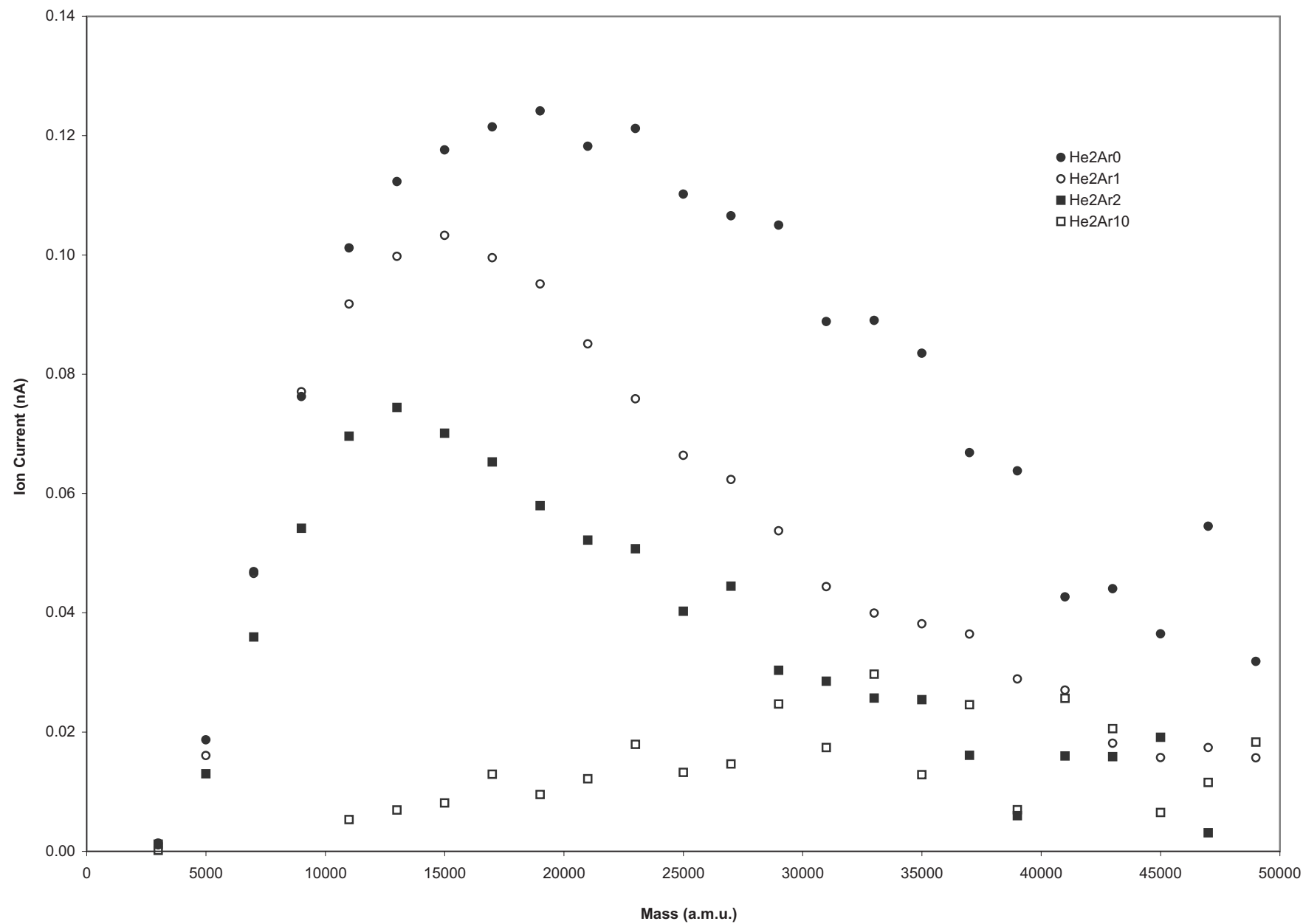

FIG. 2. Mass spectra of Fe clusters according to changes to the bath gas, P1, in terms of total pressure and ratio of He to Ar. The values given in the legend refer to mbar pressure.

sure is not linear, as shown in Fig. 3. For a given power to the crucible, a significantly higher bath gas pressure or a more efficient thermal gas will lead to some cooling of the crucible and hence a drop in flux. Ar, as a heavier gas than $\mathrm{He}$, is more efficient at condensing the metal vapor producing fewer but heavier clusters. A cooler crucible will require a higher power to evaporate at the same rate. Ar is also a good transporter of heat so the amount of heat lost to the chamber walls is higher than with He.

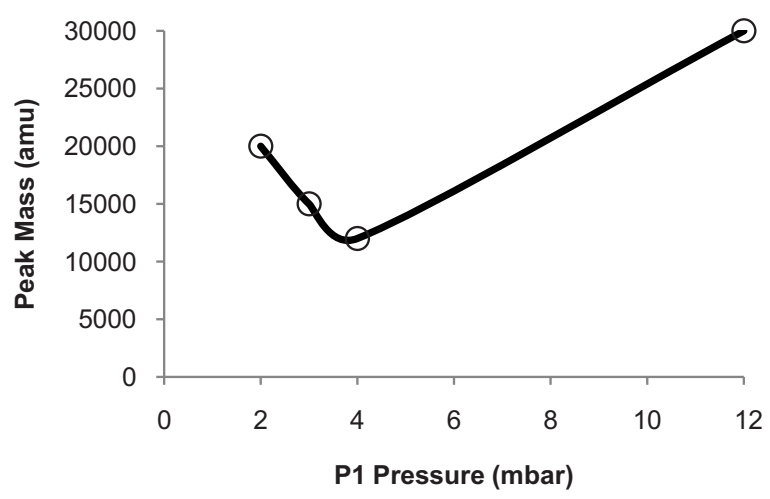

FIG. 3. Ratio of $\operatorname{He}(g)$ to $\operatorname{Ar}(g)$ and resulting affect on peak in the mass distribution of Fe clusters. The solid line serves as an aid to the eye.

\section{B. Introducing a coating gas}

A gas feed was incorporated into the main chamber after the skimmer in order to coat the clusters. By monitoring the pressure, $\mathrm{P} 3$, in the main chamber it is possible to control the amount of gas coating the clusters. Two separate studies were made using coating gases of $\mathrm{H}_{2}(g)$ and $\mathrm{N}_{2}(g)$. Figure 4 shows the mass spectra for (a) pure Fe clusters in the absence of coating gases and (b) Fe clusters for which a partial pressure of $1 \times 10^{-5}$ mbar $\mathrm{N}_{2}(\mathrm{~g})$ was added. Addition of the gas coating has shifted the mass distribution to higher masses by an amount approximately equal to a monolayer of $\mathrm{N}_{2}(g)$ and reduced the total flux of nanoparticles.

The pure Fe clusters have a peak in the mass spectrum at $15000 \mathrm{amu}$ whereas addition of the gas has shifted this peak to $19000 \mathrm{amu}$. The increase of $4000 \mathrm{amu}$ is equivalent to $\sim 290$ nitrogen atoms. A monolayer coverage on the surface of the clusters consists of $500 \pm 100$ atoms so the gas coating is approximately one layer thick. The diameter of the pure $\mathrm{Fe}$ clusters can be calculated to be $1.82 \pm 0.15 \mathrm{~nm}$ while the sample with a nitrogen shell would have a total diameter of $1.94 \pm 0.15 \mathrm{~nm}$.

It is another asset of the GAS that it can be used to prepare monolayer coatings on the clusters in this way. In order to take mass spectra measurements while using $\mathrm{H}_{2}(g)$, 


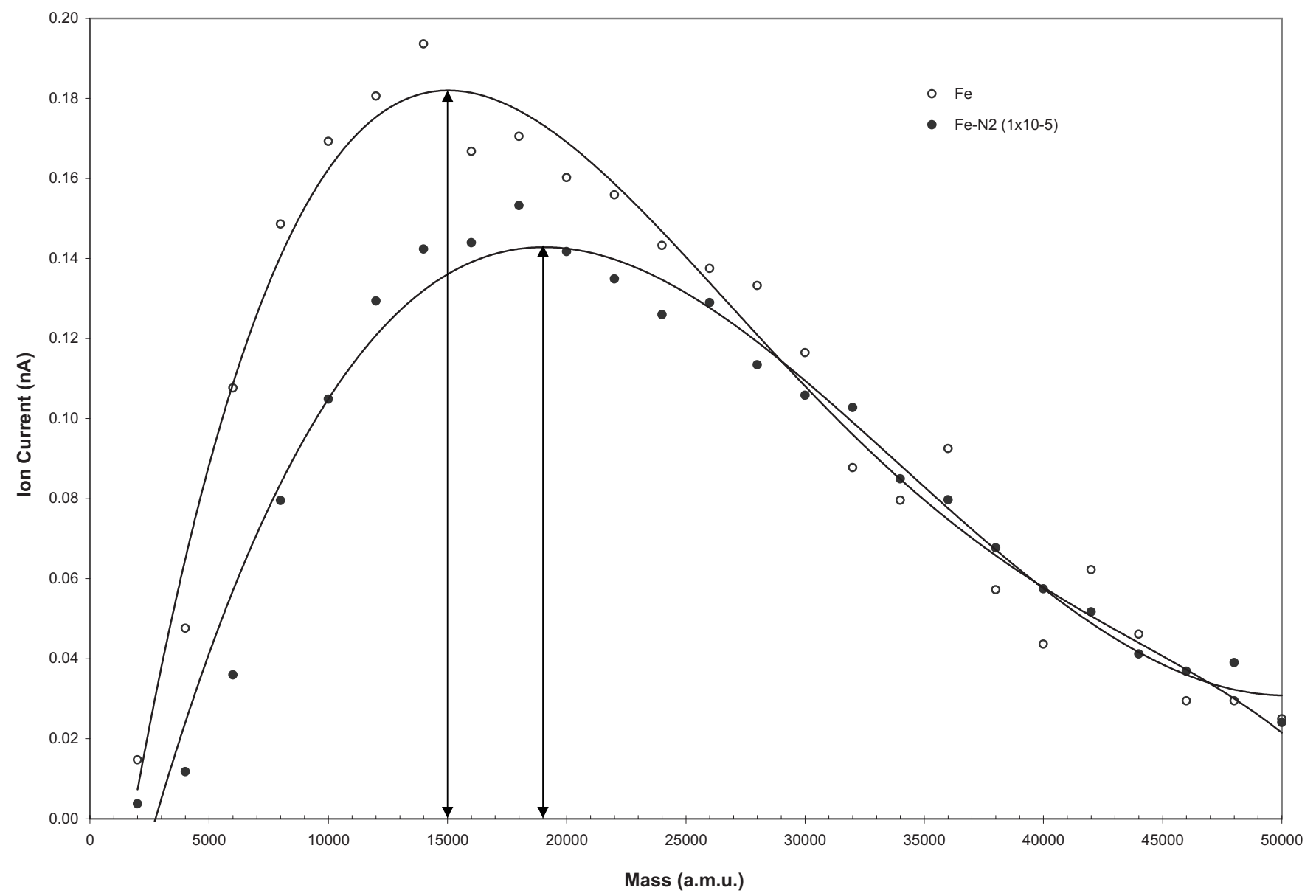

FIG. 4. Mass spectra of Fe clusters showing increase in peak mass after addition of a monolayer of $\mathrm{N}_{2}(g)$. The solid lines serve as an aid to the eye.

nonoxidizing thoriated $\mathrm{W}$ filaments had to be used in the ion source within the quadrupole. Ordinary W filaments are destroyed by the highly reactive hydrogen gas.

\section{Effect of altering crucible position}

We also investigated the effect on cluster size distributions of changing the position of the crucible within the aggregation region. Mass spectra were measured as the position of the crucible relative to the first aperture was changed. Movement was along the $y$-axis according to a geometry with the origin at the center of the first aperture. Five separate positions were studied and the resulting mass spectra obtained are summarized in Table II. Figure 5 shows the mass spectra of the Fe clusters obtained at positions (1), (2), and (5) as indicated in Table II and Fig. 6. The data clearly shows that increasing the distance from crucible to aperture results in the formation of larger clusters. For a total distance of $632 \pm 5 \mathrm{~mm}$ clusters as large as $\sim 5700$ atoms $(320000 \mathrm{amu})$ are formed. This indicates that the clusters continue to grow beyond the immediate vicinity above the crucible. This is in accordance with Robinson's inequality which states that particle densities or concentrations tend to increase along particle trajectories in incompressible irrotational flows.

\section{Production of Fe clusters in Dy matrices}

In order to demonstrate the cleanliness of the source we used it to prepare films of Fe clusters embedded in highly reactive Dy matrices by codepositing the Fe clusters and Dy from a conventional MBE source onto a common substrate mounted in an UHV chamber, which is a particularly difficult system to prepare free of contamination. Films were pro-

TABLE II. Position of crucible during reconfiguration measurements.

\begin{tabular}{llccccccc}
\hline \hline & & & & & $z$ & $\begin{array}{c}\text { Peak mass } \\
(\mathrm{amu})\end{array}$ & $\begin{array}{c}\text { Range } \\
(\mathrm{amu})\end{array}$ & Flux \\
\hline 1 & Position & Lenses & $x(\mathrm{~mm})$ & $y(\mathrm{~mm})$ & $(\mathrm{mm})$ & 20000 & 50000 & Normal \\
2 & Initial & $\mathrm{Y}$ & 0 & 302 & 0 & 15000 & 60000 & High \\
3 & Migher & $\mathrm{Y}$ & 0 & 302 & 15 & 10000 & 50000 & Low \\
4 & Middle & $\mathrm{N}$ & 0 & 100 & 0 & 28000 & 50000 & Normal \\
5 & Farture & $\mathrm{N}$ & 0 & 10 & 0 & 70000 & 320000 & High \\
\hline \hline
\end{tabular}




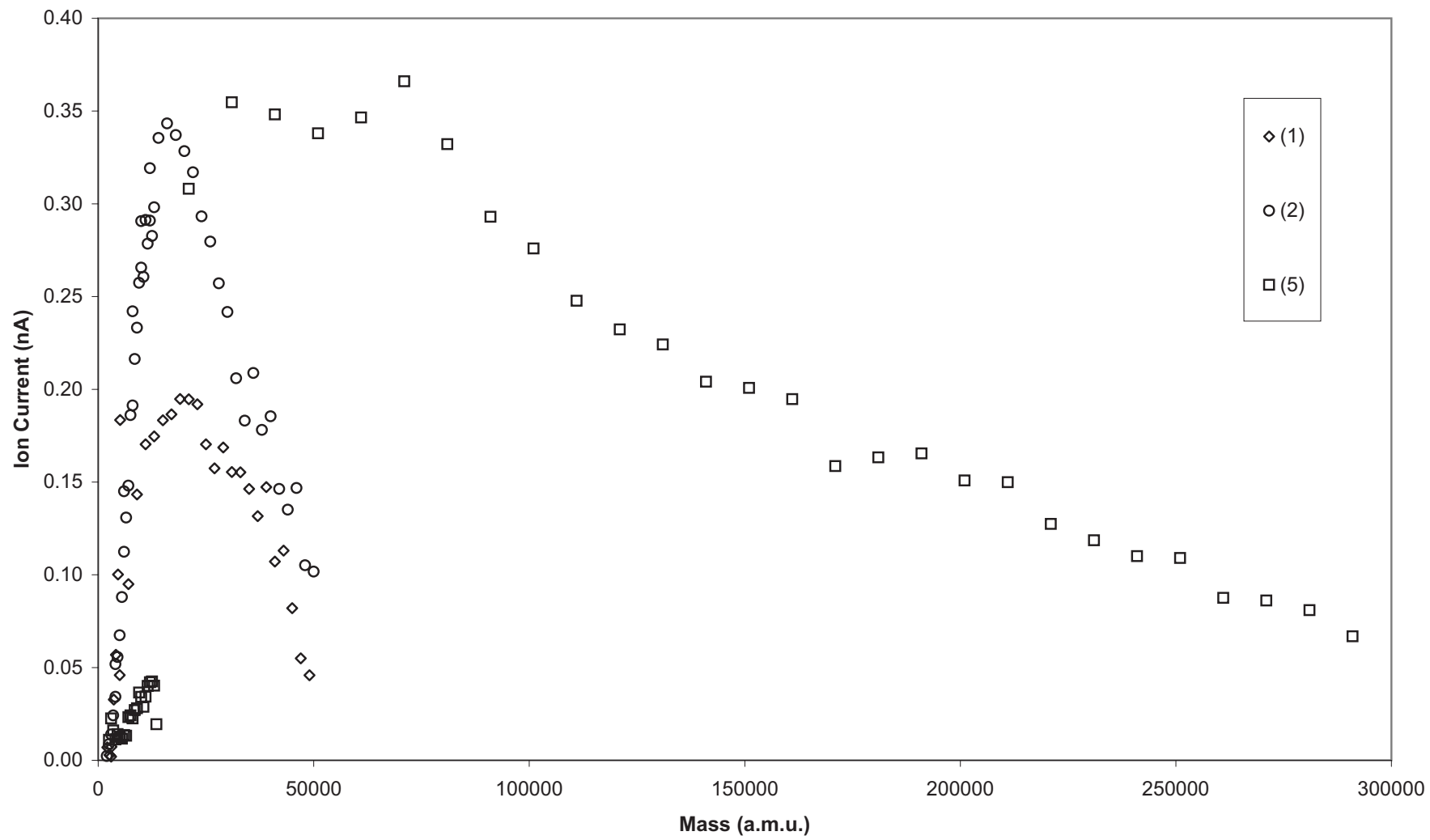

FIG. 5. Mass distribution of clusters depending on crucible position within the aggregation area. Legend refers to positions as indicated in Table II and Fig. 6.

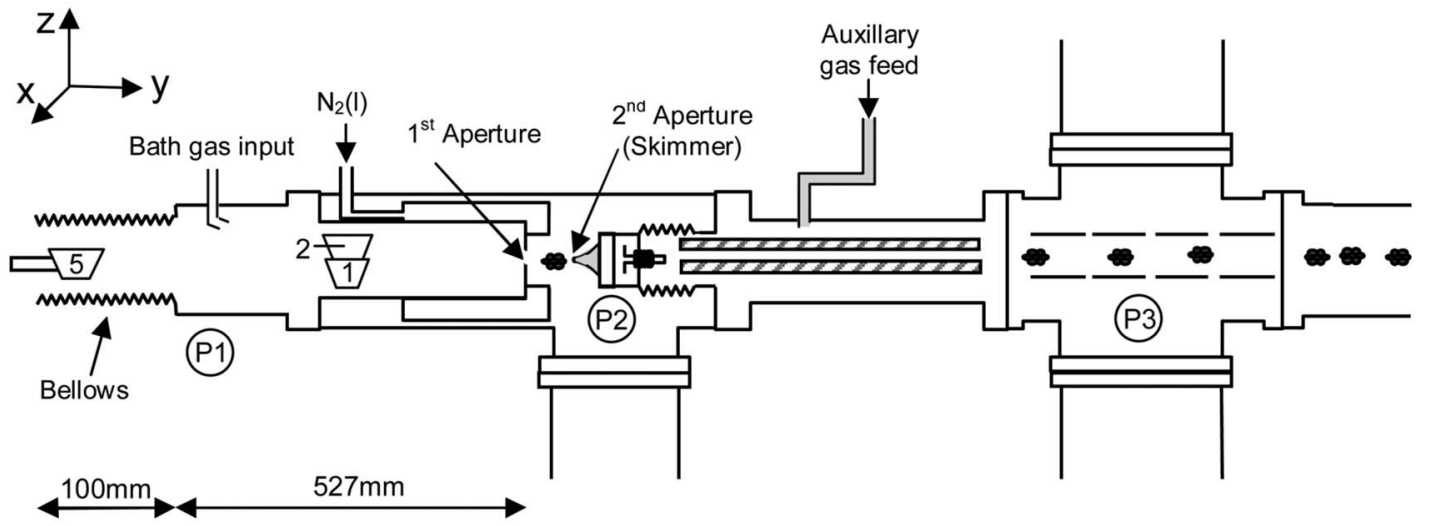

FIG. 6. GAS characterization. With the lenses removed, the crucible is free to be moved within the aggregation area. A manipulator with bellows provides a $100 \mathrm{~mm}$ extension to the overall chamber length.

TABLE III. Sample details of clean nanoparticulate samples grown using the improved GAS.

\begin{tabular}{llllll}
\hline \hline Sample & Substrate & Buffer & Matrix & v.f.f. Fe (\%) & Cap \\
\hline VSM & PEEK & $16 \mathrm{~nm} \mathrm{Ag}$ & $300 \mathrm{~nm} \mathrm{Dy}$ & $8.5 \%$ & $20 \mathrm{~nm} \mathrm{Ag}$ \\
EXAFS & Si (100) & $25 \mathrm{~nm} \mathrm{Ag}$ & $376 \mathrm{~nm} \mathrm{Dy}$ & $6.7 \%$ & $41 \mathrm{~nm} \mathrm{Ag}$ \\
\hline \hline
\end{tabular}




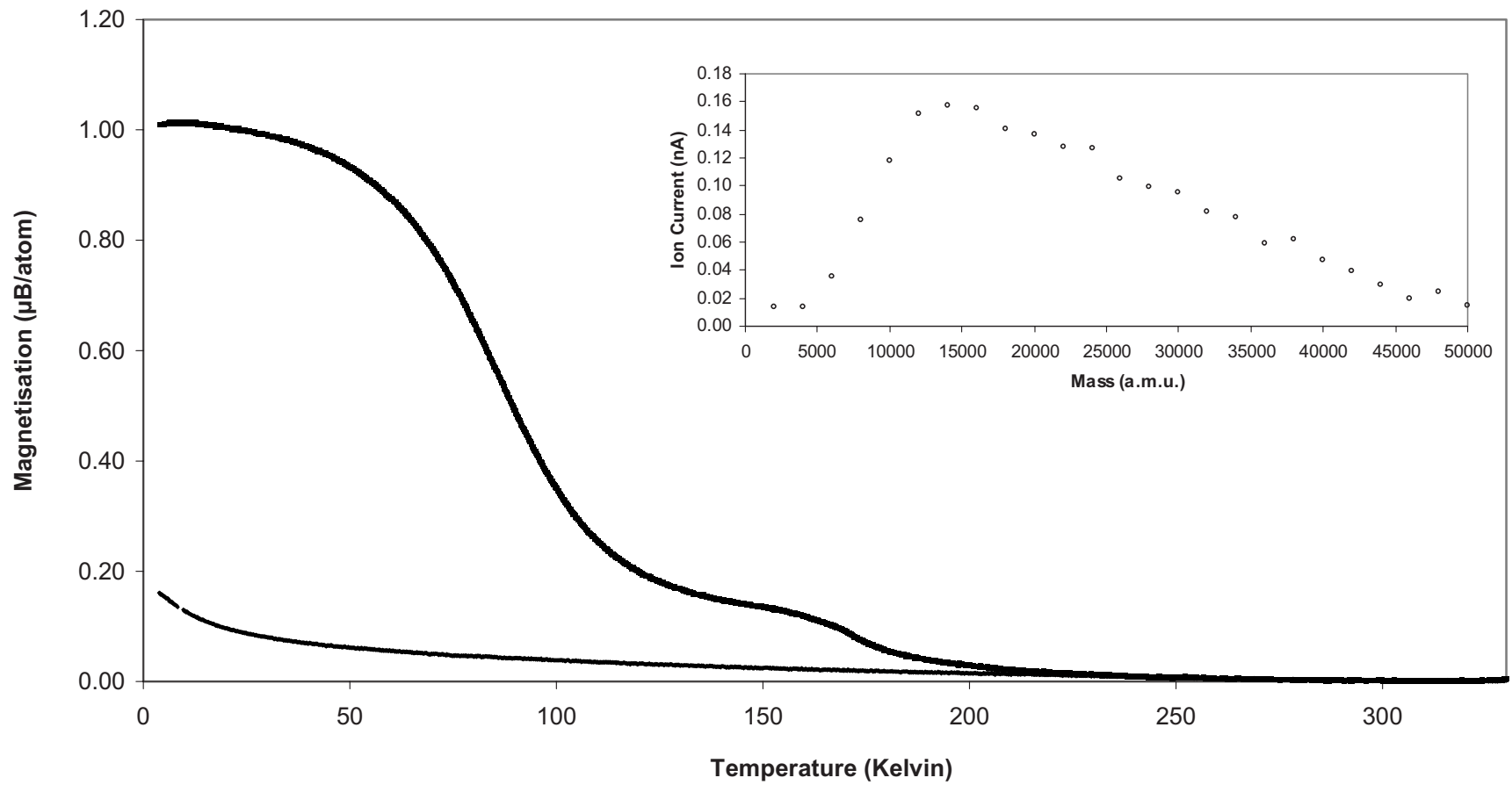

FIG. 7. The field-cooled magnetization curve for a polycrystalline sample of pure Dy (upper curve) clearly showing $T_{N}$ and $T_{C}$. The lower curve represents Fe nanoparticles in Dy (associated mass spectra as inset) showing a shift directly from paramagnetism to ferromagnetism. Both samples contain about $300 \mathrm{~nm}$ of Dy (deposited under UHV conditions) and in both cases the vibrating sample magnenometer (VSM) data were recorded using a field of $0.1 \mathrm{~T}$.

duced for magnetometry measurements on Polyetheretherketone (PEEK) substrates $25 \mu \mathrm{m}$ in thickness. An $\sim 20 \mathrm{~nm} \mathrm{Ag}$ buffer was deposited by MBE onto the substrate, while the volume filling fraction (v.f.f.) of Fe was determined by deposition rate using a XTM. The extended x-ray absorption fine structure (EXAFS) sample was prepared under similar conditions on a silicon substrate. Full sample details are given in Table III.

Fe $K$-edge EXAFS measurements reported elsewhere ${ }^{20}$ show no detectable oxidation in the embedded Fe clusters. The data are consistent, however, with a degree of alloying between the Fe and Dy.

The field-cooled magnetization curve (Fig. 7) for a polycrystalline sample of pure Dy clearly shows $T_{N}$ and $T_{C}$ consistent with clean Dy. Dysprosium oxide is paramagnetic and would not show these features. Note that the magnetization of Dy is much lower than the saturation value ( $\sim 10 \mu_{B} /$ atom) due to the small applied field. Field cooling in very high fields produces the expected magnetization for a polycrystalline film but does not show the antiferromagnetic phase, again, as expected. Inclusion of the Fe clusters drastically reduces the magnetization partly because the $\mathrm{Fe} 3 d$ moments and Dy $4 f$ moments are aligned antiparallel but also due to the formation of a high concentration Fe-Dy alloy as revealed by the EXAFS data. All these features are reported and discussed elsewhere. ${ }^{20}$

\section{CONCLUSIONS}

The versatile thermal gas aggregation source discussed here can produce metal clusters with a range of sizes and is capable of mass selection for cluster sizes from 3000 up to $320000 \mathrm{amu}$. In particular, the pressure and composition of the bath gases, as well as the position of the crucible within the source, all affect the size distribution and flux of clusters produced. In addition, the clusters can be coated with monolayers of gases, for example, nitrogen and hydrogen. These samples are all produced under UHV using highly reactive metals; measurements obtained using various experimental techniques have shown that the samples remain clean after exposure to air. The ability to produce clean samples, tailor the size of clusters produced, and have the provision to coat the surface of the clusters with a gaseous monolayer, are valuable assets in the drive to develop new cluster-assembled materials.

\section{ACKNOWLEDGMENTS}

We gratefully acknowledge support from TDK Corporation, Japan for this work.

${ }^{1}$ K. Wegner, P. Piseri, H. Vahedi Tafreshi, and P. Milani, J. Phys. D 39, R439 (2006).

${ }^{2}$ A. Perez, P. Melinon, V. Dupuis, P. Jensen, B. Prevel, J. Tuaillon, L. Bardotti, C. Martet, M. Treilleux, M. Broyen, M. Pellarin, J. L. Vialle, B. Palpant, and J. Lerme, J. Phys. D: Appl. Phys. 30, 709 (1997).

${ }^{3}$ C. Binns, K. N. Trohidou, J. Bansman, S. H. Baker, J. A. Blackman, J. P. Bucher, D. Kechrakos, A. Kleibert, S. Louch, K.-H. Meiwes-Broer, G. M. Pastor, A. Perez, and Y. Xie, J. Phys. D: Appl. Phys. 38, R357 (2005).

${ }^{4}$ H. Haberland, M. Karrais, M. Mall, and Y. Thurner, J. Vac. Sci. Technol. A 10, 3266 (1992).

${ }^{5}$ H. Haberland, M. Mall, M. Moseler, Y. Qiang, T. Reiners, and Y. Thurner, J. Vac. Sci. Technol. A 12, 2925 (1994).

${ }^{6}$ E. Barborini, P. Piseri, and P. Milani, J. Phys. D: Appl. Phys. 32, L105 (1999).

${ }^{7}$ S. Maruyama, L. R. Anderson, and R. E. Smalley, Rev. Sci. Instrum. 61, 3686 (1990).

${ }^{8}$ P. Milani and W. A. deHeer, Rev. Sci. Instrum. 61, 1835 (1990).

${ }^{9}$ H. R. Siekmann, Ch. . Luder, J. Faehrmann, H. O. Lutz, and K. H. Meiwes-Broer, Z. Phys. D: At., Mol. Clusters 20, 417 (1991).

${ }^{10}$ G. Ganteför, H. R. Siekmann, H. O. Lutz, and K. H. Meiwes-Broer, Chem. Phys. Lett. 165, 293 (1990). 
${ }^{11}$ R. P. Methling, V. Senz, D. Klinkenberg, T. Diederich, J. Tiggesbaumker, G. Holzhuter, J. Bansmann, and K. H. Meiwes-Broer, Eur. Phys. J. D 16, 173 (2001).

${ }^{12}$ K. Sattler, J. Mhlback, and E. Recknagel, Phys. Rev. Lett. 45, 821 (1980).

${ }^{13}$ S. H. Baker, S. C. Thornton, K. W. Edmonds, M. J. Maher, C. Norris, and C. Binns, Rev. Sci. Instrum. 71, 3178 (2000).

${ }^{14} \mathrm{P}$. Milani and S. Iannotta, Cluster Beam Synthesis of Nanostructured Materials (Springer, Berlin, 1999).

${ }^{15}$ P. Piseri, H. Vahedi Tafreshi, and P. Milani, Curr. Opin. Solid State Mater. Sci. 8, 192 (2004).

${ }^{16}$ X. Wang, F. E. Kruis, and P. H. McMurry, Aerosol Sci. Technol. 39, 611 (2005).

${ }^{17}$ F. Di Fonzo, A. Gidwani, M. H. Fan, D. Neumann, D. I. Iordanaglou, J. V. R. Heberlein, P. H. McMurray, S. L. Girshick, N. Tymiak, W. W. Gerberich, and N. P. Rao, Appl. Phys. Lett. 77, 910 (2000).
${ }^{18}$ K. W. Edmonds, C. Binns, S. H. Baker, M. J. Maher, S. C. Thornton, O. Tjernberg, and N. B. Brookes, J. Magn. Magn. Mater. 231, 113 (2001).

${ }^{19}$ K. W. Edmonds, C. Binns, S. H. Baker, S. C. Thornton, C. Norris, J. B. Goedkoop, M. Finazzi, and N. B. Brookes, Phys. Rev. B 60, 1 (1999).

${ }^{20}$ G. N. Iles, C. Binns, S. H. Baker, and M. Roy (submitted).

${ }^{21}$ P. Liu, P. J. Ziemann, D. B. Kittelson, and P. H. McMurray, Aerosol Sci. Technol. 22, 293 (1995).

${ }^{22}$ P. Liu, P. J. Ziemann, D. B. Kittelson, and P. H. McMurray, Aerosol Sci. Technol. 22, 314 (1995)

${ }^{23}$ D. K. Saha, K. Koga, and H. Takeo, Eur. Phys. J. D 9, 539 (1999).

${ }^{24}$ G. Palasantzas, S. A. Koch, T. Vystavel, and J. Th. M. De Hosson, J. Alloys Compd. 449, 237 (2008).

${ }^{25}$ A. Robinson, Commun. Pure Appl. Math. 9, 69 (1956).

${ }^{26}$ S. Thornton, M.S. dissertation, University of Leicester. 